\title{
Atomic-Resolution Differential Phase Contrast Imaging by STEM
}

\author{
N. Shibata, $* * *$ S.D. Findlay* and Y. Ikuhara***** \\ * Institute of Engineering Innovation, The University of Tokyo, Tokyo 113-8656 Japan. \\ ** PRESTO, Japan Science and Technology Agency, Saitama 332-0012, Japan. \\ *** Nanostructures Research Laboratory, Japan Fine Ceramic Center, Nagoya, 456-8587, Japan.
}

In scanning transmission electron microscopy (STEM), a finely focused electron probe is scanned across the specimen and the transmitted and/or scattered electrons from a localized material volume are detected by the post specimen detector(s) as a function of raster position. By controlling the detector geometry, we have a lot of flexibility in determining the contrast characteristics of STEM images and the formation mechanisms involved. Recently, we have developed a new area detector which we refer to as the "Segmented Annular All Field (SAAF)" detector and which is capable of atomic-resolution STEM imaging [1]. This area detector can obtain 16 simultaneous atomicresolution STEM images which are sensitive to the spatial distribution of scattered electrons on the detector plane [1]. The angle range can be easily controlled by changing camera length settings. Fig. 1 shows a schematic illustration of the SAAF detector. The detector has in-plane rotation capability which enables arbitrary alignment of the detector geometry to the crystallographic orientation of the sample.

With this detector, we can obtain annular bright-field and dark-field images by combining the individual STEM images from the different detector segments. Furthermore, if we take the difference between the images from diagonally opposed detector segments, we can form what are known as differential phase contrast (DPC) images [2]. It has been reported that, to a good approximation, DPC STEM images represent the gradient of the object potential (= fields) taken in the direction of the diagonally opposed detector segments, provided the object scatters weakly [2-5]. DPC STEM has been used to image magnetic structures at medium resolution [6,7]. However, DPC STEM imaging has not been applied at atomic-resolution STEM so far.

Here, we show atomic-resolution DPC STEM images of $\mathrm{SrTiO}_{3}$ observed from the [001] direction [8]. Fig. 2(a) shows the orientation relationship between the $\mathrm{SrTiO}_{3}$ crystal and the detector segments used in this study. The probe-forming aperture angle was $23 \mathrm{mrad}$ and the angle range of the detector segments was 15.3 to 30.6 mrad. Fig. 2(b) shows the experimental difference image and its intensity profile projected over the vertical direction in the image. The simultaneous ADF STEM image and its intensity profile are used for reference since the peaks in ADF image are a wellestablished indicator of the true atomic positions. It is clear that the DPC STEM profile has a node (zero crossing) at the atom location. The profile is antisymmetric about this point, reflecting the reversal of the electric field direction across the atom along the direction of diagonal detector segments. Combined with detailed image simulations, atomic-resolution DPC STEM is found to provide information on the local electric field distribution in the vicinity of the atomic columns. Some application results of atomic-resolution DPC STEM imaging will be presented.

References

[1] N. Shibata et al., J. Electron Microscopy 59, 473 (2010).

[2] N.H. Dekkers and H. de Lang, Optik, 41, 452 (1974). 
[3] H. Rose, Ultramicroscopy, 2, 251 (1977).

[4] W.C. Stewart, J. Opt. Soc. Am., 66, 813 (1976).

[5] J.M. Cowley, Ultramicroscopy, 49, 4 (1993).

[6] J.N. Chapman, J. Phys. D: Appl. Phys., 17, 623 (1984).

[7] J.N. Chapman, I. R. McFadyen, S. McVitie, IEEE Trans. Mag., 26, 1506 (1990).

[8] N. Shibata et al., submitted.

[9] This work was supported in part by the Industrial Technology Research Grant program in 2007, New Energy and Industrial Technology Development Organization (NEDO) of Japan and the Japan Society for the Promotion of Science (JSPS) through its "Funding Program for WorldLeading Innovative R\&D on Science and Technology (FIRST Program)".

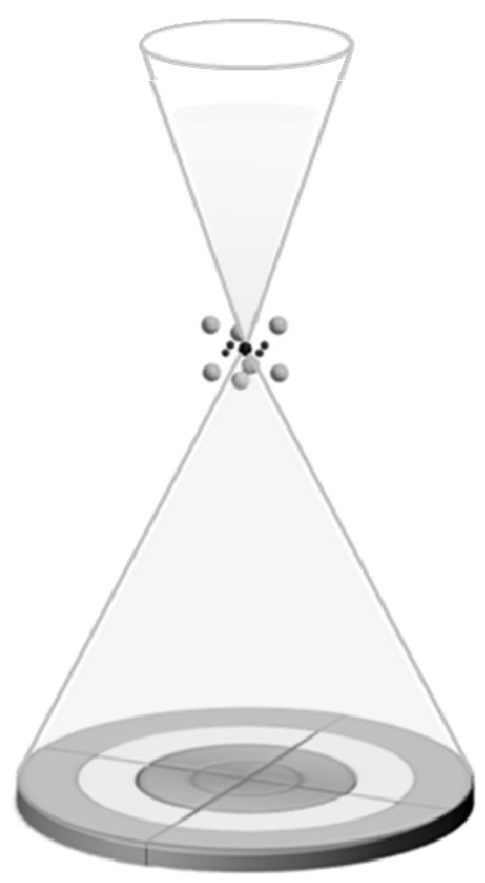

FIG. 1. Schematic of the SAAF detector. (a)

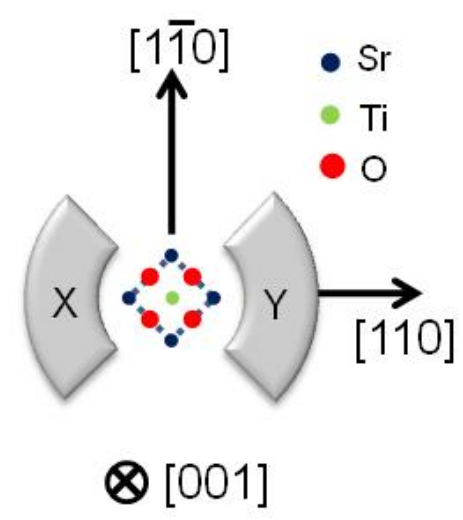

(b)
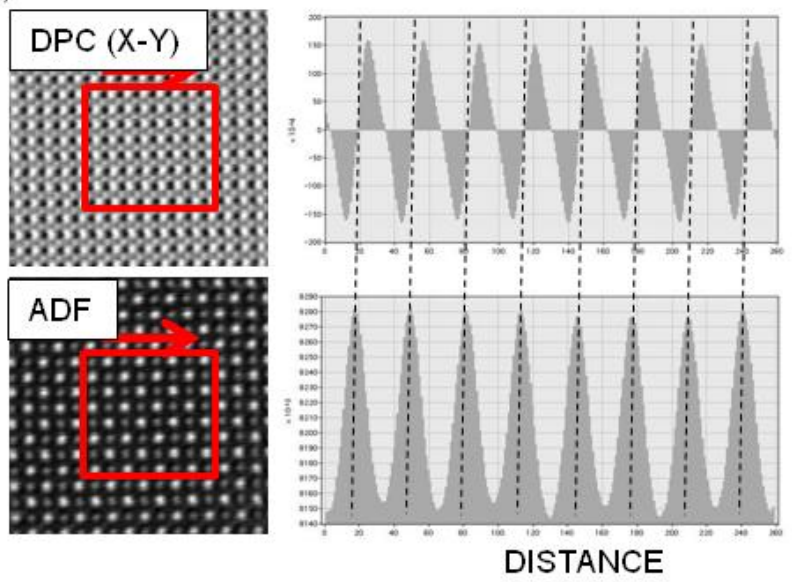

FIG. 2. (a) Schematic illustration showing the relationship between the crystallographic orientation of $\mathrm{SrTiO}_{3}$ and the two detector segments. (b) The DPC STEM image formed by subtracting the signal in detector segment $\mathrm{Y}$ from that in detector segment $\mathrm{X}$ and its image intensity profile. The simultaneous ADF image and its image intensity profile are also shown for comparison. 